Recibido: 23-04-2014

Aceptado: 20-08-2014

Palabras Clave: Motivación de logro y aprendizaje organizacional.

Keywords: Achievement motivation and organizational learning.

Julyssa Llave Zañartu

UAP julyssallz@yahoo.es

\section{La motivación de logro y el aprendizaje organizacional en los docentes de una universidad privada de lima metropolitana*}

\section{Achievement motivation and organizational learning in a professor of a private university in metropolitan Lima}

\section{Julyssa Llave Zañartu}

RESUMEN. Se pretende determinar la problemática de la motivación de logro y el aprendizaje organizacional considerando sus niveles de interacción y variaciones de a acuerdo indicadores (área de profesión, el grado académico, la edad, el lugar o lugares de trabajo, y género) correspondientes a docentes de una universidad privada de Lima. Esta investigación es explicativa y descriptiva, no experimental, transversal y correlacional, y analiza los niveles de logro y aprendizaje organizacional en los docentes y el tipo de relación existente entre la motivación de logro y el aprendizaje organizacional, así como también identificó las diferencias correspondientes a estas variables.

Los resultados indican quela motivación delogrointeractúa significativamente en el aprendizaje organizacional, así mismo que los docentes presentan diferencias significativas tanto en los niveles de motivación de logro y aprendizaje organizacional, como en sus indicadores correspondientes.

Finalmente los resultados fueron analizados y se construyó un marco doctrinario gracias a los aportes de Tapia, Montero y Huertas (2000) y Garzón y Fisher (2010), entre otros.

ABSTRACT. The problem of achievement motivation and organizational learning is going to be determined by considering their levels of interaction and variations according to respective indicators (professional field, academic degree, age, place or places of employment, and gender) corresponding to professors of a private university in Lima. This explanatory and descriptive, non-experimental, transversal, and correlational research, analyzes levels of achievement and organizational learning in professors and the type of relationship between achievement motivation and organizational learning, as well as identifies the respective differences to these variables.

The results show that achievement motivation significantly interacts in organizational learning, morever that professors have significant differences both in the levels of achievement's motivation and organizational learning as in their respective indicators.

Finally the results were analyzed and a doctrinal framework was built thanks to contributions of Tapia, Montero and Huertas (2000) and Garzon and Fisher (2010), among others.

\footnotetext{
* Tesis para optar el Grado de Doctora en Educación, UNIFE, 2014
} 


\section{Introducción}

$\mathbf{E}$ n la actualidad, las instituciones de nivel universitario tienen como objetivo principal brindar un servicio educativo de calidad. En esa intencionalidad, dentro de un contexto competitivo, el conocimiento del potencial humano es el capital intangible de mayor trascendencia para la organización educativa. En ese orden de ideas, la motivación de logro del potencial humano garantiza no solamente un servicio educativo de calidad que facilita el aprendizaje de los educandos; sino, también, garantiza un aprendizaje organizacional.

El objetivo de la investigación fue determinar la problemática de la motivación de logro y el aprendizaje organizacional en términos de sus características, niveles de interacción, y variaciones de acuerdo indicadores (área de profesión, el grado académico, la edad, el lugar o lugares de trabajo y género) de los docentes de una universidad privada de Lima, basada en la doctrina de Tapia, Montero y Huertas (2000), Garzón y Fisher (2010).

La investigación presenta el planteamiento del problema que responde a la siguiente pregunta: ¿De qué manera la motivación de logro y el aprendizaje organizacional interactúan en los docentes de una universidad privada de Lima?. Se tomó como unidad de análisis las encuestas tomadas a docentes de la Facultad de Administración y Negocios Internacionales de una Universidad privada de Lima. Asimismo, presenta el marco teórico de cada una de las variables que proporciona el sustento del presente trabajo, específicamente centrado en la doctrina antes mencionada. Finalmente, presenta el sistema de hipótesis y el diseño metodológico propio de una investigación tipo básica, sustantiva. Según el enfoque cuantitativo, es un estudio descriptivo y explicativo aplicándose la estadística para la recopilación de los datos contando con una muestra de cincuenta docentes. Se encontró que los docentes con motivación de logro no solamente mejoran eficientemente su aprendizaje organizacional, sino también elevan los niveles de la calidad educativa; asimismo, se observan diferencias tanto en el aprendizaje organizacional como en la
Alétheia 2014, 2 (1) 69

motivación de logro de acuerdo a indicadores (área de profesión, el grado académico, la edad, el lugar o lugares de trabajo y género). En ese orden de ideas, se presentan los resultados, la discusión de los resultados y por último las conclusiones y las respectivas referencias bibliográficas.

\section{Materiales y Método}

\section{Materiales}

El material de trabajó fue:

1. Cuestionario Motivación de Logro: MAPE-3. Tapia, Montero y Huertas (2000).

2. Cuestionario de Aprendizaje Organizacional. Garzón y Fischér (2010).

\section{Método}

El nivel de investigación es básica y sustantiva, porque se encontraron interacciones entre motivación de logro y del aprendizaje organizacional en los docentes de una universidad privada de Lima.

De acuerdo al enfoque cuantitativo, el tipo de investigación es un estudio descriptivo - explicativo. Sánchez y Reyes (1985), sostienen que es descriptivo porque a partir de una muestra probabilística de docentes de la Escuela de Administración y Negocios Internacionales de una universidad privada, se descubrieron características de motivación de logro y aprendizaje organizacional y se comprobaron la asociación entre las variables de la población. En la investigación descriptiva se recolecta información relevante en muestras con respecto a un mismo fenómeno, en base a la comparación de los datos recopilados, es posible realizar una comparación entre ellos.

Bernal (2002), señala que la investigación explicativa es el tipo de investigación más usada en las Ciencias Sociales. Cuando en una investigación el investigador plantea como objetivo estudiar el porqué de las cosas, hechos, fenómenos o situaciones a estas investigaciones se les denomina explicativa. Cuanto mayor número de variables estén correlacionadas 


\section{UNIFÉ - EPG}

en el estudio y mayor será la fuerza de las relaciones, más completa será la explicación, según Hernández, Fernández y Baptista (1996). Por lo expuesto la investigación es explicativa porque se determinó las características de los docentes para medir su motivación de logro y ver cómo explica el comportamiento del aprendizaje organizacional de los docentes.

El diseño de la investigación es no experimental, transversal y correlacional, ya que la investigación asocia la motivación de logro con el aprendizaje organizacional para establecer comparaciones. Se aplicó los instrumentos en un solo tiempo a efectos de establecer la relación entre motivación de logro y aprendizaje organizacional de los docentes.

El procedimiento usado fue el siguiente:

\section{Primero}

Determinación de las variables, las dimensiones. * Variable Independiente

"Motivación de Logro"

Dimensiones:

a) Motivación por la tarea

b) Motivación Extrínseca

c) Ansiedad facilitadora del rendimiento

* Variable Dependiente

"Aprendizaje organizacional"

Factores:

a) Condiciones para el aprendizaje organizacional

b) Cultura para el aprendizaje organizacional

c) Fuentes del aprendizaje organizacional

Segundo

Determinación de sujetos a estudiar:

La investigación tiene una muestra de cincuenta docentes de la Facultad de Administración y Negocios Internacionales de una Universidad privada de Lima de los turnos mañana, tarde y noche.

\section{RESULTADOS}

Tabla 1

Correlación entre motivación de logro y aprendizaje organizacional de los docentes de una Universidad Privada.

Aprendizaje

Organizacional

Motivación de logro

Correlación de Pearson

$0.857^{\star}$

Significancia bilateral

0.000

Tamaño de muestra

50

* La correlación es significativa al nivel 0.01 (bilateral).

La hipótesis general establecía que existe interacción significativa entre la motivación de logro y el aprendizaje organizacional de los docentes de una universidad privada de Lima. Los resultados presentados en la tabla 1 indican una correlación directa positiva y significativa $(r=0.857, p<0.01)$ entre motivación de logro y aprendizaje organizacional.

Tabla 2

Niveles de motivación de logro de los docentes de una universidad Privada.

\begin{tabular}{ccccc}
\hline Niveles & Total & Porcentaje & $\begin{array}{c}\text { Estadistica } \\
\text { de Pruevba }\end{array}$ & $\mathrm{p}$ \\
\hline Medio & 14 & 28 & & \\
Alto & 36 & 72 & $\mathrm{X}^{2}=9.68^{*}$ & 0.002 \\
Total & 50 & 100 & g.l. $=1$ & \\
\hline F $<0.05$. & 1-25: Muy Bajo, 26-50: & Bajo, 51-75: Medio, 76-100: \\
Alto & \multicolumn{4}{l}{} \\
\end{tabular}

De acuerdo a los resultados, la sub hipótesis 1.1 que sostenía que los docentes presentaban diferencias significativas en los niveles de motivación de logro ha sido confirmada y se observan niveles adecuados. 
Tabla 3

Niveles de aprendizaje organizacional de los docentes de una Universidad Privada.

\begin{tabular}{ccccc}
\hline Niveles & Total & Porcentaje & $\begin{array}{c}\text { Estadistica } \\
\text { de Pruevba }\end{array}$ & $\mathrm{p}$ \\
\hline Medio & 8 & 16 & & \\
Alto & 42 & 84 & $\mathrm{X}^{2}=9.68^{*}$ & 0.000 \\
Total & 50 & 100 & g.l. $=1$ & \\
\hline * $<$ <.05. & 1-25: Muy Bajo, 26-50: & Bajo, 51-75: Medio, 76-100: \\
Alto & & & &
\end{tabular}

Los resultados presentados en la tabla 3 permiten observar que el nivel de aprendizaje organizacional en la muestra estudiada el cual en el $84 \%$ de los casos es de nivel alto, seguido por el nivel medio en el 16\% de los casos. Se observan niveles adecuados.

Tabla 4

Nivel de aprendizaje organizacional según género, de los docentes de una Universidad Privada.

\begin{tabular}{ccccc}
\hline \multirow{2}{*}{ Género } & & \multicolumn{2}{c}{ Niveles de aprendizaje } & Estadistica de \\
Prueba
\end{tabular}

${ }^{*} \mathrm{p}<0.05$. 1-25: Muy Bajo, 26-50: Bajo, 51-75: Medio, 76-100: Alto

En la tabla 4 se observan diferencias significativas en los docentes con niveles medio y alto. Por otro lado, como se puede apreciar, las docentes presentan un nivel mucho más alto de aprendizaje organizacional en comparación con los niveles de aprendizaje organizacional de los docentes.

\section{Discusión de Resultados}

\section{Relación entre Motivación de Logro y Aprendizaje Organizacional}

Para Valle et al., (2010), la motivación fortalece el proceso de aprendizaje: de ahí la importancia de desarrollar estrategias motivacionales para consolidar una conciencia motivacional positiva orientada al logro del aprendizaje organizacional.

Coincidentemente, Calero (2009) señala que la falta de motivación conlleva al fracaso del aprendizaje; asimismo, destaca los aportes del actor educativo, así como también las cualidades como la conversación, buena escucha, respeto, confianza que deben tener, a fin de conocer las condiciones que favorecen tanto la motivación como el aprendizaje organizacional. En consecuencia, los actores sociales, en general deben reconocer la importancia de la motivación de logro y sus efectos positivos en el aprendizaje organizacional. Ciertamente, en la práctica organizacional, los gerentes educativos motivan en sentido extrínseco dejando de lado la motivación de logro.

En cuanto a la motivación de logro, los resultados ubicados en la tabla 1 indican que existe una correlación significativa, muy fuerte y positiva con el aprendizaje organizacional, lo cual fue evaluado mediante el coeficiente de correlación de Pearson, que permite analizar el grado de relación existente entre las variables. En consecuencia, a medida que un docente aumenta su puntaje de motivación de logro aumenta su aprendizaje organizacional. Lo que implica que los docentes con motivación de logro tienen desarrollada tanto la competencia para lograr un aprendizaje organizacional; así como también la competencia de activar emociones positivas, fortalecer las debilidades académicas, repotenciar capacidades y conocimientos previos de los estudiantes en el proceso educativo.

\section{Diferencias en los niveles de Motivación de Logro y Aprendizaje Organizacional}

Con respecto a la primera subhipótesis: Los docentes de una universidad privada de Lima, presentan diferencias significativas en los niveles de motivación de logro, se encontró que existen diferencias significativas en los puntajes de motivación de logro (Tabla 2), los resultados validados a través de la prueba Chi-cuadrada, a fin de analizar las diferencias existentes en los niveles, muestran el predominio del nivel alto de motivación de logro. Al respecto, Luna 


\section{UNIFÉ - EPG}

(2003) manifiesta que los factores de la Motivación de Logro se refieren al crecimiento personal, pasión por el trabajo, responsabilidad social, oportunidad de avanzar, el sentimiento de realización o de logro, ventajas propias y a destacar positivamente. En ese sentido, los gestores de conocimiento de cada organización deben tener como misión permanente controlar los factores y diseñar estrategias que permitan el logro de la visión de la organización.

Bellido (2005), reafirma lo anterior cuando opina que el potencial humano tiene la capacidad para automotivarse y el líder la capacidad de reconocer las cualidades, convencer, convertir a su potencial en generadores del conocimiento y finalmente encaminarlo hacia su realización profesional $\mathrm{y}$ personal. Por su parte, Calero (2009) concluye que la falta de motivación constituye una causa que deteriora el aprendizaje. Asimismo, plantea una propuesta donde destaca la importancia que tiene el conocimiento de los factores que favorecen la motivación; que de tenerlos en cuenta, la organización se convertiría en un verdadero pilar de construcción del conocimiento, tal como lo plantea en su obra: "Aprendizaje sin límites".

Los estudios citados permiten afirmar que la motivación tiene significatividad respecto al aprendizaje. En ese sentido, la motivación constituye una gran herramienta educativa cuya aplicación vence tanto a la ansiedad, al miedo al fracaso y al desinterés por el trabajo. Así como también, refuerza el aprendizaje, autoestima, deseo de tener éxito y de disfrutar cada reto en aplicación de distintos valores que orientan la vida académica y laboral, tal como lo señalan Tapia, Montero y Huertas (2000), cuando destacan los factores de la motivación en su obra : "Evaluación de la motivación en sujetos adultos".

El alto nivel de motivación de logro de los docentes, puede ser explicada por el hecho de que los docentes tienen un estado consiente positivo, pues se trata de profesionales que han definido sus objetivos y se orientan a lograrlas a través de la enseñanza universitaria, porque cuentan con herramientas que facilitan el proceso educativo tales como: la conversación y motivación.
Con respecto a la tercera subhipótesis: Los docentes de una universidad privada de Lima, presentan diferencias significativas en los niveles de aprendizaje organizacional. Los resultados (Tabla 3), muestran el predominio del nivel alto de aprendizaje organizacional seguido del nivel medio en los docentes, diferencias significativas observadas a través de la prueba Chi-cuadrada con la finalidad de analizar las diferencias existentes en los niveles. Lieuri y Fenoíuillet (2006), señalan que el aprendizaje es producto de la motivación y del grado de conocimiento, en consecuencia existe una correlación fuerte entre la motivación y el aprendizaje.

En la muestra, el predominio del nivel alto de aprendizaje organizacional en los docentes de una universidad privada de Lima, indica que los docentes pueden convertir en práctica de vida la visión trazada por la universidad y asimismo estarían capacitados para formar educandos autónomos, porque cuentan con recursos como el liderazgo.

\section{Aprendizaje Organizacional de los docentes según indicador "género"}

Las docentes presentan un nivel alto de aprendizaje organizacional (Tabla 4). Díaz (2004), destaca en su obra "Liderazgo de la mujer gerente en Universidades y sus implicaciones para el aprendizaje organizacional", la participación activa de la mujer en el ámbito educativo: "En Venezuela las mujeres participan activamente en organizaciones civiles y en el ámbito laboral, destacándose en el sistema educativo (Universidades), donde ejercen cargos de mediana y alta gerencia" (p. 67).

Por otro lado, el autor describe situación social de la mujer, en el ámbito laboral. En esta investigación, se estudia una élite femenina, mujeres que van a la cabeza, desenvolviéndose en terrenos difíciles, competitivos, como la Educación Superior, pese a la serie de mecanismos legales que propugnan su equidad e igualdad formal. Esta élite representa la imagen social dominante de lo femenino, de la mujer profesional, del valor social, que han vencido los obstáculos socioculturales profundos que obstruyen 
la consecución por parte de las mujeres de puestos de poder y reparto más equilibrado por género del mismo, convirtiéndose, además estas mujeres élites, en un modelo y ejemplo de la posibilidad de alcanzar el éxito para otras mujeres (Díaz, 2004, p. 72).

Finalmente, el autor plantea las conclusiones de su hallazgo:

Las lideresas investigadas desarrollaron estrategias de gestión exitosas, tales como trabajo en equipo, procesos administrativos que permitieran cambios y uso eficiente, eficaz y pertinente de los recursos, procesos de comunicación basados en el diálogo y la apertura, perseverancia, superación, relaciones políticas, sustentadas en el apoyo de las bases y en el reconocimiento de los méritos propios y de sus colaboradores. Todo esto les permitió propiciar en las organizaciones por ellas gerenciadas un impacto en los procesos de aprendizaje organizacional (Díaz, 2004, p. 75).

Por consiguiente, las explicaciones del autor demuestran que la participación de la mujer en una universidad constituye una ventaja competitiva. Finalmente, su prestación de servicios garantiza tanto un aprendizaje organizacional, como el cumplimiento efectivo de la misión de la Universidad. Así, los resultados de la presente investigación, confirman doctrina del autor: Las docentes tienen un alto nivel de aprendizaje organizacional.

\section{Conclusiones}

Luego de realizado el análisis y discusión de los resultados en esta investigación, se presenta las conclusiones que se desprende de la importancia del docente, en las aulas, sus factores de motivación de logro que transmite a sus discentes y su aprendizaje organizacional que desarrolla dentro de una organización educativa.

1. Entre las variables estudiadas se observa una correlación alta y positiva entre aprendizaje organizacional y motivación de logro, lo que indica que a medida que un docente aumente su motivación de logro, aumentará asimismo su aprendizaje organizacional:
2. Los docentes presentan adecuados niveles de motivación de logro y aprendizaje organizacional. En ese sentido, los docentes ven reflejada su visión personal en la visión institucional, se identifican y muestran una actitud positiva hacia la misma en términos de motivación y productividad lo que le permite crear entornos de aprendizajes significativos en busca de la calidad educativa.

3. Se destaca entre los docentes, una coexistencia de variaciones existentes respecto de su motivación delogro según los siguientes indicadores: área de profesión, grado académico, edad, trabaja además en otras instituciones y género.

\section{Referencias}

Bellido, J. (2005). Motivar en el aula: El arte de hacer que hagan. Málaga, España: Aljibe.

Bernal, C. (2000). Metodología de la Investigación p a r a Administración y Economía. Bogotá, Colombia: Prentince.

Calero, M. (2009). Aprendizaje sin limites. Constructivismo. México, D.F., México: Alfa Omega.

Díaz,M. (2004). Liderazgo de la mujergerente en Universidades y sus implicaciones para el aprendizaje organizacional. Venezolana de Estudios de la mujer, 9, 67-77. Recuperado de http://sa ber. ucv.ve/ojs/index. $\mathrm{php} / \mathrm{rev}$ vem/article/view/2256/2149

Garzón, A., y Fischer, A. (2010). El aprendizaje organizacional, prueba piloto de instrumentos tipo Likert. Empresa, 15, 65 - 101. Recuperado de http://redalyc. uaemex.mx/src/ini cio/ ArtPdfRed .jsp? iCve $=631151230$

Hernández. R., Fernández. C., y Baptista, P. (1996). Metodología de la Investigación. México, D. F., México: Mc. Graw Hill.

Lieuri,A.,y Fenouillet, F.(2006). Motivación y Éxito Escolar: México, D.F., México: Fondo de Cultura Económica.

Luna, R. (2003). El papel de la motivación financiera y la motivación de logro en la dirección estratégica de recursos humanos. Dirección y Economía de la Empresa, 12, pp. 205-216. 
74 UNIFÉ - EPG

Recuperado de http://dialnet.unirioja.es/ servlet/articulo?codigo $=638919$

Sánchez, C., y Reyes, M. (1985). Metodología y Diseños en la Investigación Científica. Lima. Perú: Mantaro.

Tapia, A., Montero, I., y Huertas, J. (2000). Evaluación de la motivación en sujetos adultos. Universidad Autónoma de Madrid, 5, 1-29. Recuperado en http://sohs.pbs.uam.es/webjesus /eval_ psicologica/cuestionarios/mape3. pdf

Valle, A., Rodríguez, S., Núñez, J., Cabanach, R., González - Pienda,J., y Rosario, P. (2010). Motivación y Aprendizaje Autorregulado. Invenio, 44, 86- 97. Recuperado de http:// redalyc.uaemex.mx/redaly c/src/inicio/ ArtPdfRed.jsp?iCve $=28420600040010$ 